\title{
Population specific validation of measures of bone mineral content from bioelectric impedance analyser: comparison with dual energy x-ray absorptiometer
}

\author{
O. Okantey, E. Amoako Atta and S. R. Mitra \\ School of Human Sciences, London Metropolitan University, London
}

Up to $90 \%$ of peak bone mass in the body is gained by age of 18 in females and 20 in males, which makes youth the best time to "invest" in bone health ${ }^{(1)}$. Measuring Bone mineral content (BMC) during early life can help prevent degeneration of bone micro-architecture in later life with early effective action ${ }^{(2)}$. Studies indicate that bioelectric impedance analysis (BIA) shows good agreement with dual energy $\mathrm{x}$-ray absorptiometry (DEXA) on measures of fat mass, percent fat and fat-free mass in different populations ${ }^{(3)}$ and has potential for widespread use because of simplicity and portability. Thus it is of great clinical interest to know how well Tanita performs in assessing total body bone mineral content in human subjects.

This study will assess the validity of the Tanita BC-418 for measurements of appendicular BMC in British-African children and young adults through comparison with DEXA.

British-African subjects aged 4-19 years were measured. All segmental BIA measurements were performed by using a single frequency bioimpedance analyzer (BC-418 MA, Tanita Corp., Tokyo, Japan). BMC measures from bioelectrical impedance analysis were arrived at by subtracting the predicted muscle mass (PMM) from the fat-free mass (FFM). Whole body scans were performed on the same subjects using Norland XR800 Dual-Energy X-Ray Absorptiometer (DEXA) as the reference method. Methods used to assess agreement were Bland-Altman pair-wise comparisons and Pearson's correlation coefficient. Ethical approval was granted by the London Metropolitan University Research Ethics Review Panel.

In total 20 females and 24 males were measured. Mean ages were 11.0 y (S.E. 3.42) in females and 10.08 y (S.E. 4.39) in males. BMD $\left(\mathrm{g} / \mathrm{cm}^{2}\right)$ ranged from 0.56-1.22 in females and 0.63-1.08 males. Total BMC $(\mathrm{g})$ ranged from 782.4-3259.0 and 838.5-3138.0 respectively. Tanita showed excellent agreement with DEXA for appendicular BMC.

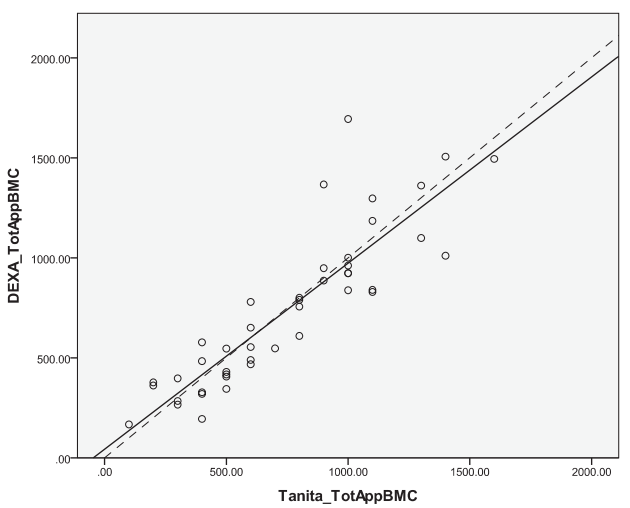

It tends to underestimate total appendicular BMC in subjects with BMC below $600 \mathrm{~g}$ and overestimates in subjects with BMC above $600 \mathrm{~g}$ (dotted line represents $\mathrm{y}=\mathrm{x}$; fig. A). In the combined data Tanita overestimated by just $1.2 \%$ o(Bland-Altman pairwise comparison; fig. B). There was an error of overestimation of $15.3 \%$ in girls and underestimation of $-10.5 \%$ in boys. This information could validate the use of this simple technique in assessing bone health for preliminary screening in primary health care settings or epidemiological research in community setting.

1. Ho AY \& Kung AW (2005) Determinants of Peak Bone Mineral Density and Bone Area in Young Women. J Bone Miner Metab 23, 470-5.

2. Bedogni G, Mussi C, Malavolti M, Borghi A, Poli M, Battistini N \& Salvioli G (2002) Relationship between Body Composition and Bone Mineral Content in Young and Elderly Women. Annals of Human Biology 29.5, 559-65.

3. Sluyter JD, Schaaf D, Scragg RK \& Plank LD (2010) Prediction of fatness by standing 8-electrode bioimpedance: a multiethnic adolescent population Obesity 18(1): 183-9. 